\title{
A Left and Right Truncated Lognormal Distribution for the Stars
}

\author{
L. Zaninetti \\ Physics Department, via P.Giuria 1, I-10125 Turin,Italy \\ Email: zaninetti@ph.unito.it
}

\begin{abstract}
The initial mass function for the stars is often modeled by a lognormal distribution. This paper is devoted to demonstrating the advantage of introducing a left and right truncated lognormal probability density function, which is characterized by four parameters. Its normalization constant, mean, the variance, second moment about the origin and distribution function are calculated. The chi-square test and the Kolmogorov-Smirnov test are performed on four samples of stars.
\end{abstract}

Keywords: Stars, characteristics and properties of Stars, normal

\section{Introduction}

The initial mass function (IMF) for the stars was firstly fitted with a power law by Salpeter, see [1]. He suggested $p(m) \propto m^{-\alpha}$ where $p(m)$ represents the probability of having a mass between $m$ and $m+d m$ and he found $\alpha=2.35$ in the range $10 M_{\odot}>M \geq 1 M_{\odot}$. Secondly the IMF was fitted with three power laws, see $[2,3,4]$ and four power laws, see $[5,6,7]$. The piecewise broken inverse power law IMF is

$$
p(m) \propto m^{-\alpha_{i}},
$$

each zone being characterized by a different exponent $\alpha_{i}$ and two boundaries $m_{i}$ and $m_{i+1}$. In order to have a probability density function (PDF) normalized to unity, one must have

$$
\sum_{i=1, n} \int_{m_{i}}^{m_{i+1}} c_{i} m^{-\alpha_{i}} \mathrm{~d} m=1
$$

The number of parameters to be found from the considered sample for the $n$-piecewise IMF is $2 n-1$ when $m_{1}$ and $m_{n+1}$ are the minimum and maximum of the masses of the sample. In the case of $n=4$, which fits also the region of brown dwarfs (BD), see [8], the number of parameters is seven. In the field of statistical distributions, the PDF is usually defined by two parameters. Examples of two-parameter PDFs are: the beta, gamma, normal, and lognormal distributions, see [9]. The lognormal distribution is widely used in order to model the IMF for the stars, see $[10,11,12,13]$. The lognormal distribution is defined in the range of $\mathcal{M} \in(0, \infty)$ where $\mathcal{M}$ is the mass of the star. Nevertheless, the stars have minimum and maximum values, as an example from the MAIN SEQUENCE, an M8 star has $\mathcal{M}=0.06 \mathcal{M} \odot$ and an O3 star has $\mathcal{M}=120 \mathcal{M}_{\odot}$, see [14]. The presence of boundaries for the stars makes attractive the analysis of a left and right truncated lognormal. In Section 2, the structure of the lognormal distribution is reviewed. In Section 3, the truncated lognormal distribution is derived. In Section 4.2, a comparison between the lognormal and truncated lognormal is done on four catalogs of stars. In Section 5, we compare the results of the truncated lognormal distribution with the double Pareto lognormal, the truncated beta, and the truncated gamma distributions.

\section{The Lognormal Distribution}

Let $X$ be a random variable defined in $[0, \infty]$; the lognormal $\mathrm{PDF}$, following [9] or formula $(14.2)^{\prime}$ in [15], is

$$
P D F(x ; m, \sigma)=\frac{\mathrm{e}^{-\frac{1}{2 \sigma^{2}}\left(\ln \left(\frac{x}{m}\right)\right)^{2}}}{x \sigma \sqrt{2 \pi}},
$$


where $m$ is the median and $\sigma$ the shape parameter. The distribution function (DF) is

$$
D F(x ; m, \sigma)=\frac{1}{2}+\frac{1}{2} \operatorname{erf}\left(\frac{1}{2} \frac{\sqrt{2}(-\ln (m)+\ln (x))}{\sigma}\right),
$$

where $\operatorname{erf}(\mathrm{x})$ is the error function, defined as

$$
\operatorname{erf}(x)=\frac{2}{\sqrt{\pi}} \int_{0}^{x} e^{-t^{2}} \mathrm{~d} t
$$

see [16]. The average value or mean, $E(X)$, is

$$
E(X ; m, \sigma)=m \mathrm{e}^{\frac{1}{2} \sigma^{2}},
$$

the variance, $\operatorname{Var}(X)$, is

$$
\operatorname{Var}=\mathrm{e}^{\sigma^{2}}\left(\mathrm{e}^{\sigma^{2}}-1\right) m^{2},
$$

the second moment about the origin, $E^{2}(X)$, is

$$
E\left(X^{2} ; m, \sigma\right)=m^{2} \mathrm{e}^{2 \sigma^{2}} .
$$

The experimental sample consists of the data $x_{i}$ with $i$ varying between 1 and $n$; the sample mean, $\bar{x}$, is

$$
\bar{x}=\frac{1}{n} \sum_{i=1}^{n} x_{i}
$$

the unbiased sample variance, $s^{2}$, is

$$
s^{2}=\frac{1}{n-1} \sum_{i=1}^{n}\left(x_{i}-\bar{x}\right)^{2}
$$

and the sample $r$ th moment about the origin, $\bar{x}_{r}$, is

$$
\bar{x}_{r}=\frac{1}{n} \sum_{i=1}^{n}\left(x_{i}\right)^{r}
$$

The parameter estimation is here obtained in two ways. The matching moments estimator, (MME), is the first method:

$$
E(X ; m, \sigma)=\bar{x}_{1} \quad ; \quad E\left(X^{2} ; m, \sigma\right)=\bar{x}_{2} \quad
$$

and therefore

$$
\begin{aligned}
& \widehat{m}=\frac{\bar{x}_{1}^{2}}{\sqrt{\bar{x}_{2}}} \\
& \widehat{\sigma}=\sqrt{2} \sqrt{\ln \left(\frac{\sqrt{\bar{x}_{2}}}{\bar{x}_{1}}\right)} .
\end{aligned}
$$

The second method implements the maximum-likelihood estimation (MLE), see [9].

\section{The Truncated Lognormal Distribution}

Let $X$ be a random variable defined in $\left[x_{l}, x_{u}\right]$; the truncated lognormal PDF $\left(P D F_{T}\right)$ is

$$
P D F_{T}\left(x ; m, \sigma, x_{l}, x_{u}\right)=\frac{\sqrt{2} \mathrm{e}^{-\frac{1}{2} \frac{1}{\sigma^{2}}\left(\ln \left(\frac{x}{m}\right)\right)^{2}}}{-\sqrt{\pi} \sigma\left(\operatorname{erf}\left(\frac{1}{2} \frac{\sqrt{2}}{\sigma} \ln \left(\frac{x_{l}}{m}\right)\right)-\operatorname{erf}\left(\frac{1}{2} \frac{\sqrt{2}}{\sigma} \ln \left(\frac{x_{u}}{m}\right)\right)\right) x},
$$


where $m$ is now the scale parameter, $\sigma$ is the shape parameter, $x_{l}$ denotes the minimal value, and $x_{u}$ denotes the maximal value. The introduction of the following coefficients allows a compact notation

$$
\begin{aligned}
& a_{1}=\frac{1}{2} \frac{\sqrt{2}\left(-\sigma^{2}+\ln \left(x_{l}\right)-\ln (m)\right)}{\sigma}, \\
& a_{2}=\frac{1}{2} \frac{\sqrt{2}\left(\sigma^{2}+\ln (m)-\ln \left(x_{u}\right)\right)}{\sigma}, \\
& a_{3}=\frac{1}{2} \frac{\sqrt{2}\left(\ln \left(x_{l}\right)-\ln (m)\right)}{\sigma}, \\
& a_{4}=\frac{1}{2} \frac{\sqrt{2}\left(-\ln \left(x_{u}\right)+\ln (m)\right)}{\sigma}, \\
& a_{5}=\frac{1}{2} \frac{\sqrt{2}\left(-2 \sigma^{2}+\ln \left(x_{l}\right)-\ln (m)\right)}{\sigma}, \\
& a_{6}=\frac{1}{2} \frac{\sqrt{2}\left(2 \sigma^{2}+\ln (m)-\ln \left(x_{u}\right)\right)}{\sigma}, \\
& a_{7}=\frac{1}{2} \frac{\sqrt{2}\left(-2 \sigma^{2}+\ln \left(x_{u}\right)-\ln (m)\right)}{\sigma}, \\
& a_{8}=\frac{1}{2} \frac{\sqrt{2}\left(\ln \left(x_{u}\right)-\ln (m)\right)}{\sigma} .
\end{aligned}
$$

In the compact notation the PDF is

$$
P D F_{T}\left(x ; m, \sigma, x_{l}, x_{u}\right)=\frac{-\sqrt{2} \mathrm{e}^{-\frac{1}{2} \frac{1}{\sigma^{2}}\left(\ln \left(\frac{x}{m}\right)\right)^{2}}}{\sqrt{\pi} \sigma\left(\operatorname{erf}\left(a_{3}\right)-\operatorname{erf}\left(a_{8}\right)\right) x},
$$

the $\mathrm{DF}$ is

$$
D F_{T}\left(x ; m, \sigma, x_{l}, x_{u}\right)=\frac{-\operatorname{erf}\left(\frac{1}{2} \frac{\sqrt{2}}{\sigma} \ln \left(\frac{x}{m}\right)\right)+\operatorname{erf}\left(a_{3}\right)}{\operatorname{erf}\left(a_{3}\right)-\operatorname{erf}\left(a_{8}\right)},
$$

the mean, $E(X)_{T}$, is

$$
E_{T}\left(X ; m, \sigma, x_{l}, x_{u}\right)=\frac{\mathrm{e}^{\frac{1}{2} \sigma^{2}} m\left(\operatorname{erf}\left(a_{1}\right)+\operatorname{erf}\left(a_{2}\right)\right)}{\operatorname{erf}\left(a_{3}\right)+\operatorname{erf}\left(a_{4}\right)},
$$

the variance, $\operatorname{Var}_{T}(X)$, is

$$
\operatorname{Var}_{T}\left(X ; m, \sigma, x_{l}, x_{u}\right)=\frac{N}{\left(\operatorname{erf}\left(a_{3}\right)+\operatorname{erf}\left(a_{4}\right)\right)^{2}},
$$

where

$$
\begin{gathered}
\quad N=\mathrm{e}^{\sigma^{2}}\left(\operatorname{erf}\left(a_{3}\right) \operatorname{erf}\left(a_{5}\right) \mathrm{e}^{\sigma^{2}}+\operatorname{erf}\left(a_{3}\right) \operatorname{erf}\left(a_{6}\right) \mathrm{e}^{\sigma^{2}}+\operatorname{erf}\left(a_{4}\right) \operatorname{erf}\left(a_{5}\right) \mathrm{e}^{\sigma^{2}}\right. \\
\left.+\operatorname{erf}\left(a_{4}\right) \operatorname{erf}\left(a_{6}\right) \mathrm{e}^{\sigma^{2}}-\left(\operatorname{erf}\left(a_{1}\right)\right)^{2}-2 \operatorname{erf}\left(a_{1}\right) \operatorname{erf}\left(a_{2}\right)-\left(\operatorname{erf}\left(a_{2}\right)\right)^{2}\right) m^{2},
\end{gathered}
$$

the second moment about the origin, $E_{T}^{2}(X)$, is

$$
E_{T}\left(X^{2} ; m, \sigma, x_{l}, x_{u}\right)=\frac{-\mathrm{e}^{2 \sigma^{2}} m^{2}\left(-\operatorname{erf}\left(a_{5}\right)+\operatorname{erf}\left(a_{7}\right)\right)}{\operatorname{erf}\left(a_{3}\right)+\operatorname{erf}\left(a_{4}\right)} .
$$

The two parameters $x_{l}$ and $x_{u}$ are the minimal and maximal elements of the sample. The two parameters $m$ and $\sigma$ can be found through the MME, first method

$$
E_{T}\left(X ; m, \sigma, x_{l}, x_{u}\right)=\bar{x}_{1} \quad ; \quad E_{T}\left(X^{2} ; m, \sigma, x_{l}, x_{u}\right)=\bar{x}_{2} \quad .
$$

The above system consists in two non-linear functions in two variables and can therefore be solved using the Powell hybrid method, see subroutine FORTRAN SNSQE in [17]. The second method implements the MLE in order to find $m$ and $\sigma$, see Appendix 6 . 


\section{Application to the Stars}

This section reviews some useful statistical parameters, such as the merit function $\chi^{2}$, the Akaike information criterion, and the Kolmogorov-Smirnov test. The four samples of stars which test the truncated lognormal distribution are introduced.

\subsection{The Adopted Statistics}

The merit function $\chi^{2}$ is computed according to the formula

$$
\chi^{2}=\sum_{i=1}^{n} \frac{\left(T_{i}-O_{i}\right)^{2}}{T_{i}},
$$

where $n$ is the number of bins, $T_{i}$ is the theoretical value, and $O_{i}$ is the experimental value represented by the frequencies. The theoretical frequency distribution is given by

$$
T_{i}=N \Delta x_{i} p(x),
$$

where $N$ is the number of elements of the sample, $\Delta x_{i}$ is the magnitude of the size interval, and $p(x)$ is the PDF under examination. The size of the bins, $\Delta x_{i}$, is equal for each bin in the the case of linear histograms, but different for each bin when logarithmic histograms are considered.

A reduced merit function $\chi_{\text {red }}^{2}$ is evaluated by

$$
\chi_{\text {red }}^{2}=\chi^{2} / N F
$$

where $N F=n-k$ is the number of degrees of freedom, $n$ is the number of bins, and $k$ is the number of parameters. The goodness of the fit can be expressed by the probability $Q$, see equation 15.2.12 in [18], which involves the degrees of freedom and $\chi^{2}$. According to [18] p. 658, the fit "may be acceptable" if $Q>0.001$.

The Akaike information criterion (AIC), see [19], is defined by

$$
A I C=2 k-2 \ln (L),
$$

where $L$ is the likelihood function and $k$ the number of free parameters in the model. We assume a Gaussian distribution for the errors and the likelihood function can be derived from the $\chi^{2}$ statistic $L \propto \exp \left(-\frac{\chi^{2}}{2}\right)$ where $\chi^{2}$ has been computed by eqn. (23), see [20], [21]. Now the AIC becomes

$$
A I C=2 k+\chi^{2}
$$

The Kolmogorov-Smirnov test $(\mathrm{K}-\mathrm{S})$, see $[22,23,24]$, does not require binning the data. The K-S test, as implemented by the FORTRAN subroutine KSONE in [18], finds the maximum distance, $D$, between the theoretical and the astronomical DF as well the significance level $P_{K S}$, see formulas 14.3.5 and 14.3.9 in [18]; if $P_{K S} \geq 0.1$, the goodness of the fit is believable.

\subsection{The Selected Sample of Stars}

The test samples are selected from the Centre de Données astronomiques de Strasbourg (CDS) in order to ensure that the test can be easily reproduced, the name of the catalog is reported. The first test is performed on the low-mass IMF in the young cluster NGC 6611, see [25] and CDS catalog J/MNRAS/392/1034. This massive cluster has an age of $2-3 \mathrm{Myr}$ and contains masses from $1.5 M_{\odot}>M \geq 0.02 M_{\odot}$. Therefore the brown dwarfs $(\mathrm{BD})$ region, $\approx 0.2 \mathcal{M}_{\odot}$ is covered. Table 1 shows the values of $\chi_{\text {red }}^{2}$, the AIC, the probability $Q$, of the fits and the two results of the $\mathrm{K}-\mathrm{S}$ test: the maximum distance, $D$, between the theoretical and the astronomical DF as well the significance level $P_{K S}$. Figure 1 shows the fit with the truncated lognormal DF for NGC 6611, and Figure 2 the truncated lognormal PDF.

The second test is performed on NGC 2362 where the 271 stars have a range $1.47 M_{\odot}>M \geq 0.11 M_{\odot}$, see [26] and CDS catalog J/MNRAS/384/675/table1. This is a very young open cluster with an estimated 


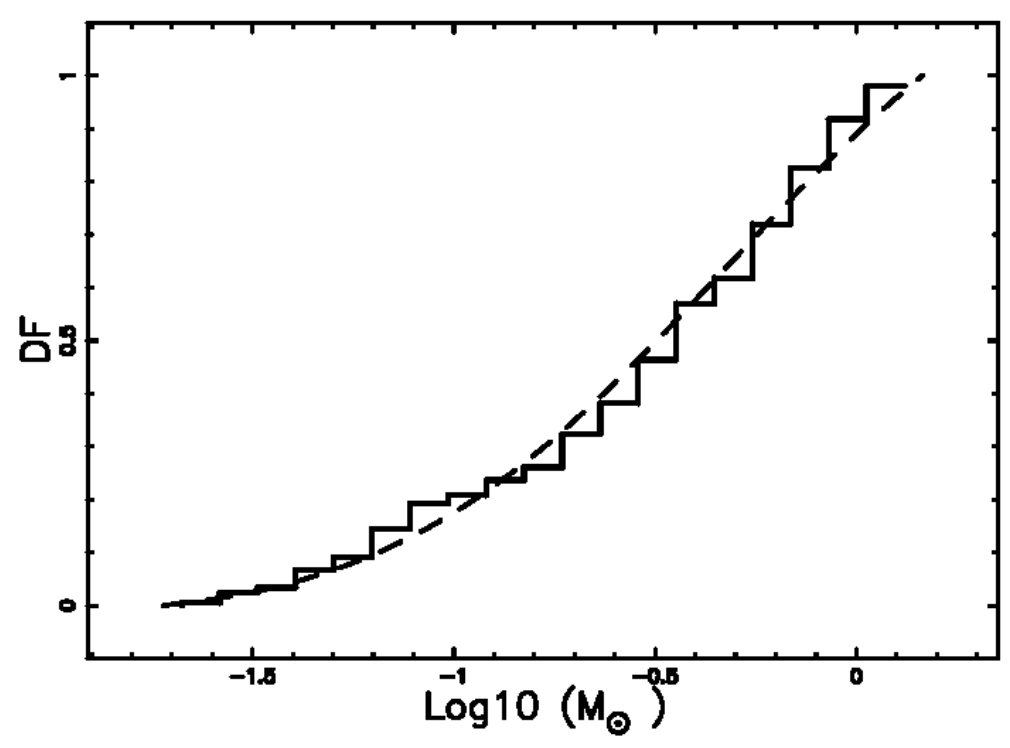

Figure 1. Empirical DF of mass distribution for NGC 6611 cluster data (207 stars + BDs) when the number of bins, $n$, is 20 (steps with full line) with a superposition of the truncated lognormal DF (dashed line). Theoretical parameters as in Table 1, MLE method. The horizontal axis has a logarithmic scale.

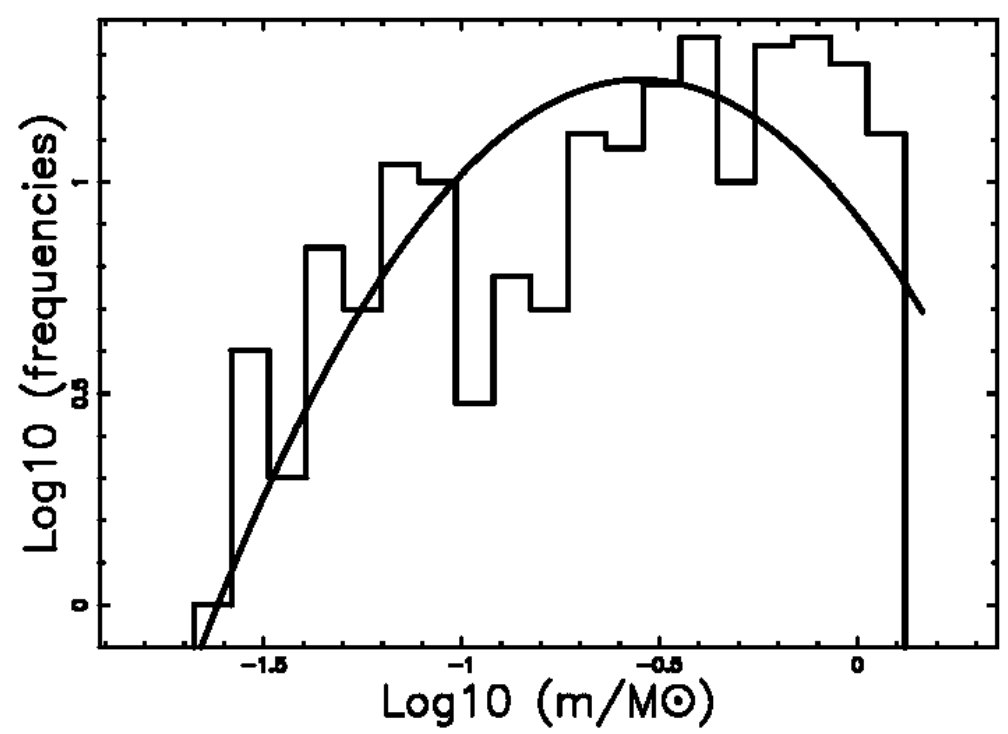

Figure 2. Frequencies of mass distribution for NGC 6611 cluster data (steps with full line) with a superposition of the truncated lognormal PDF (full line). Parameters as in Figure 1. The vertical and horizontal axes have a logarithmic scale. 


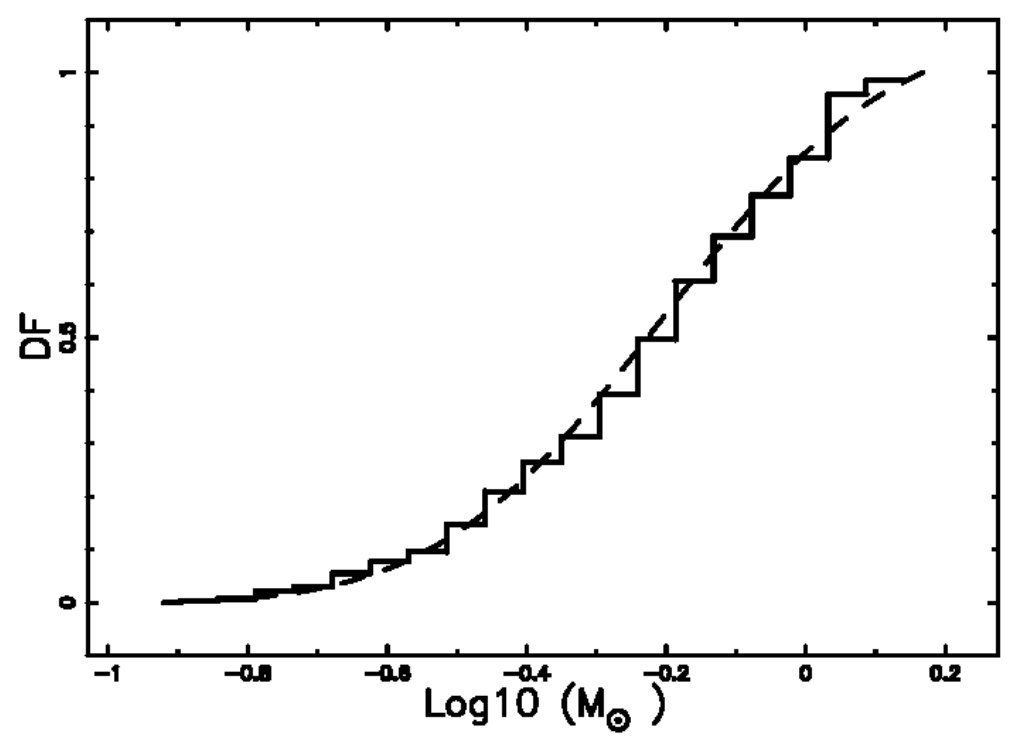

Figure 3. Empirical DF of mass distribution for NGC 2362 cluster data ( 273 stars + BDs) when the number of bins, $n$, is 20 (steps with full line) with a superposition of the truncated lognormal DF (dashed line). Theoretical parameters as in Table 2, MLE method. The horizontal axis has a logarithmic scale.

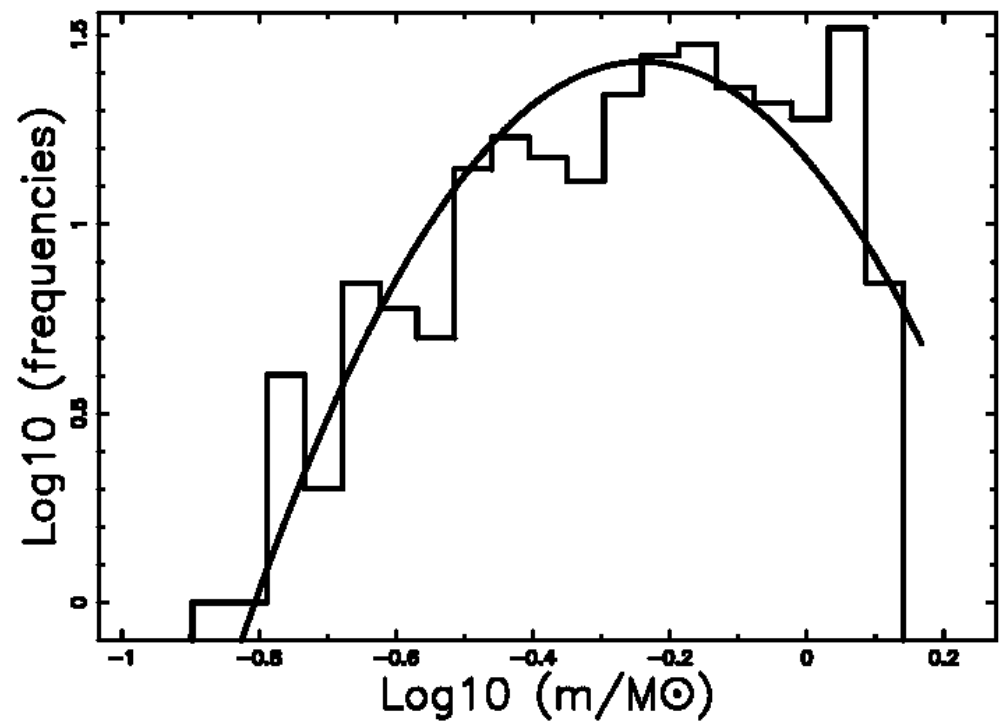

Figure 4. Frequencies of mass distribution for NGC 2362 cluster data (steps with full line) with a superposition of the truncated lognormal PDF (full line). Parameters as in Figure 3. The vertical and horizontal axes have a logarithmic scale. 
Table 1. Statistical parameters of NGC 6611 (207 stars + BDs). The number of linear bins, $n$, is 20 .

\begin{tabular}{|c|c|c|c|c|c|c|c|}
\hline PDF & Method & parameters & AIC & $\chi_{\text {red }}^{2}$ & $Q$ & D & $P_{K S}$ \\
\hline lognormal & MLE & $\sigma=1.029, m=0.284$ & 71.24 & 3.73 & $1.310^{-7}$ & 0.09366 & 0.04959 \\
\hline lognormal & MME & $\sigma=0.676, m=0.339$ & 107.46 & 5.74 & $5.110^{-14}$ & 0.172 & $6.710^{-6}$ \\
\hline truncated lognormal & MLE & $\sigma=1.499, m=0.478, x_{l}=0.0189, x_{u}=1.46$ & 50.96 & 2.68 & $2.810^{-4}$ & 0.0654 & 0.372 \\
\hline truncated lognormal & MME & $\sigma=0.977, m=0.361, x_{l}=0.0189, x_{u}=1.46$ & 71.30 & 3.95 & $1.4310^{-7}$ & 0.117 & 0.005 \\
\hline
\end{tabular}

Table 2. Statistical parameters of NGC 2362 (272 stars). The number of linear bins, $n$, is 20 .

\begin{tabular}{|c|c|c|c|c|c|c|c|}
\hline PDF & Method & parameters & AIC & $\chi_{\text {red }}^{2}$ & $Q$ & $\mathrm{D}$ & $P_{K S}$ \\
\hline lognormal & MLE & $\sigma=0.507, m=0.574$ & 37.64 & 1.86 & 0.013 & 0.072 & 0.105 \\
\hline lognormal & MME & $\sigma=0.428, m=0.588$ & 51.66 & 2.648 & $1.6810^{-4}$ & 0.0842 & 0.039 \\
\hline truncated lognormal & MLE & $\sigma=0.59, m=0.625, x_{l}=0.119, x_{u}=1.47$ & 50.498 & 2.656 & $3.3310^{-4}$ & 0.047 & 0.556 \\
\hline truncated lognormal & MME & $\sigma=0.521, m=0.612, x_{l}=0.119, x_{u}=1.47$ & 46.05 & 2.37 & $1.410^{-3}$ & 0.048 & 0.525 \\
\hline
\end{tabular}

age of 3-9 Myr. Table 2 reports the statistical parameters, Figure 3 shows the fit with the truncated lognormal DF of NGC 2362 and Figure 4 the fit with the truncated lognormal PDF.

The third test is performed on a $40^{\prime}$ circular field in the LMC made by 1563 stars in the range of masses, evaluated assuming an age of $4 \mathrm{Myr}, 54 M_{\odot}>M \geq 5 M_{\odot}$, see [27] and CDS catalog $\mathrm{J} /$ ApJ/425/122/table2. Table 3 reports the statistical parameters. Figures 5 and 6 shows the fit with the

Table 3. Statistical parameters of a circular field in the LMC (1563 stars). The number of linear bins, $n$, is 20 .

\begin{tabular}{|c|c|c|c|c|c|c|c|}
\hline PDF & Method & parameters & AIC & $\chi_{\text {red }}^{2}$ & $Q$ & $\mathrm{D}$ & $P_{K S}$ \\
\hline lognormal & MLE & $\sigma=0.533, m=13.84$ & 139.32 & 7.51 & $5.0710^{-20}$ & 0.0981 & $1.3810^{-13}$ \\
\hline lognormal & MME & $\sigma=0.554, m=13.80$ & 122.07 & 6.55 & $9.610^{-17}$ & 0.0884 & $4.0210^{-11}$ \\
\hline truncated lognormal & MLE & $\sigma=0.64, m=12.9, x_{l}=5, x_{u}=54$ & 94.90 & 5.43 & $9.2510^{-12}$ & 0.073 & $8.2410^{-8}$ \\
\hline truncated lognormal & MME & $\sigma=0.7, m=12.36, x_{l}=5, x_{u}=54$ & 102.6 & 5.91 & $3.5110^{-13}$ & 0.0895 & $2.2510^{-11}$ \\
\hline
\end{tabular}

truncated lognormal DF and PDF respectively.

The fourth test is performed on $\gamma$ Velorum cluster where the 237 stars have a range $1.31 M_{\odot}>M \geq$ $0.15 M_{\odot}$, see [28] and CDS catalog $\mathrm{J} / \mathrm{A}+\mathrm{A} / 589 / \mathrm{A} 70 /$ table5. This cluster is consists of 5-10 Myr old premain sequence stars. The statistical parameters are reported in Table 4, Figures 7 and 8 report the truncated lognormal DF and PDF respectively.

Table 4. Statistical parameters of $\gamma$ Velorum cluster (237 stars). The number of linear bins, $n$, is 20.

\begin{tabular}{|c|c|c|c|c|c|c|c|}
\hline PDF & Method & parameters & AIC & $\chi_{\text {red }}^{2}$ & $Q$ & $\mathrm{D}$ & $P_{K S}$ \\
\hline lognormal & MLE & $\sigma=0.504, m=0.337$ & 55.13 & 2.84 & $5.0810^{-5}$ & 0.0921 & 0.0334 \\
\hline lognormal & MME & $\sigma=0.564, m=0.331$ & 52.47 & 2.69 & $1.210^{-4}$ & 0.099 & 0.017 \\
\hline truncated lognormal & MLE & $\sigma=0.805, m=0.227$ & 30.54 & 1.4 & 0.126 & 0.052 & 0.509 \\
\hline truncated lognormal & MME & $\sigma=0.504, m=0.337$ & 38.1 & 2.38 & $1.410^{-3}$ & 0.131 & $4.810^{-3}$ \\
\hline
\end{tabular}

\section{Other New Distributions}

As an initial astronomical reference, we display a piecewise broken inverse power law PDF, see Figure 9 


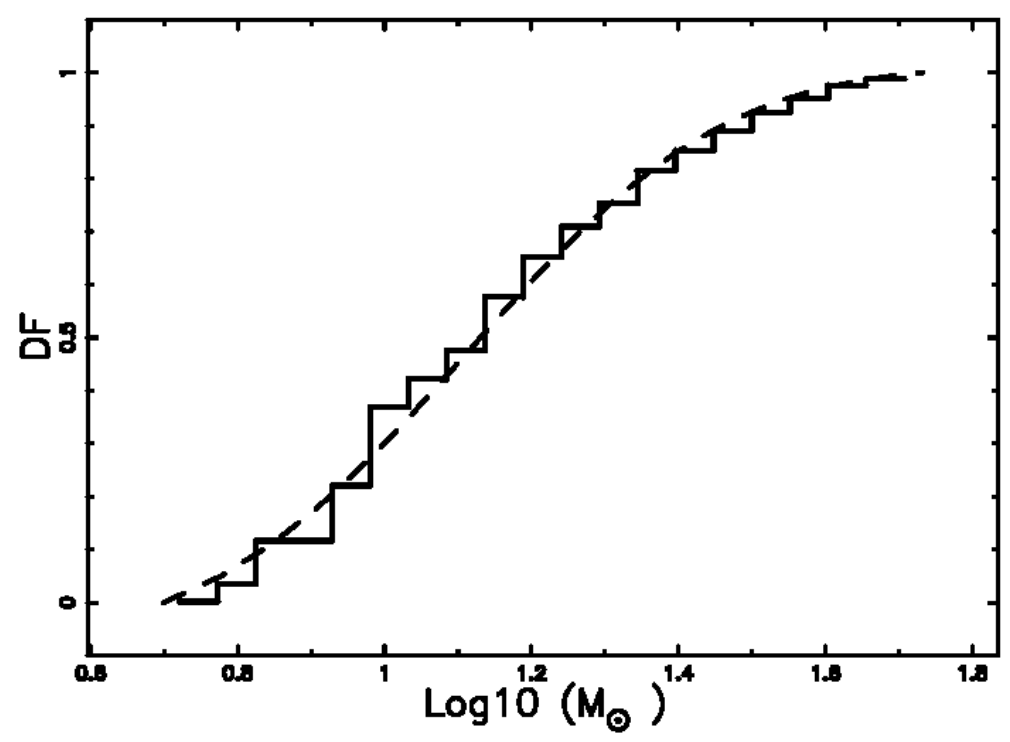

Figure 5. Empirical DF of mass distribution for 1563 stars in LMC when the number of bins, $n$, is 20 (steps with full line) with a superposition of the truncated lognormal distribution (dashed line). Theoretical parameters as in Table 3, MLE method. The horizontal axis has a logarithmic scale.

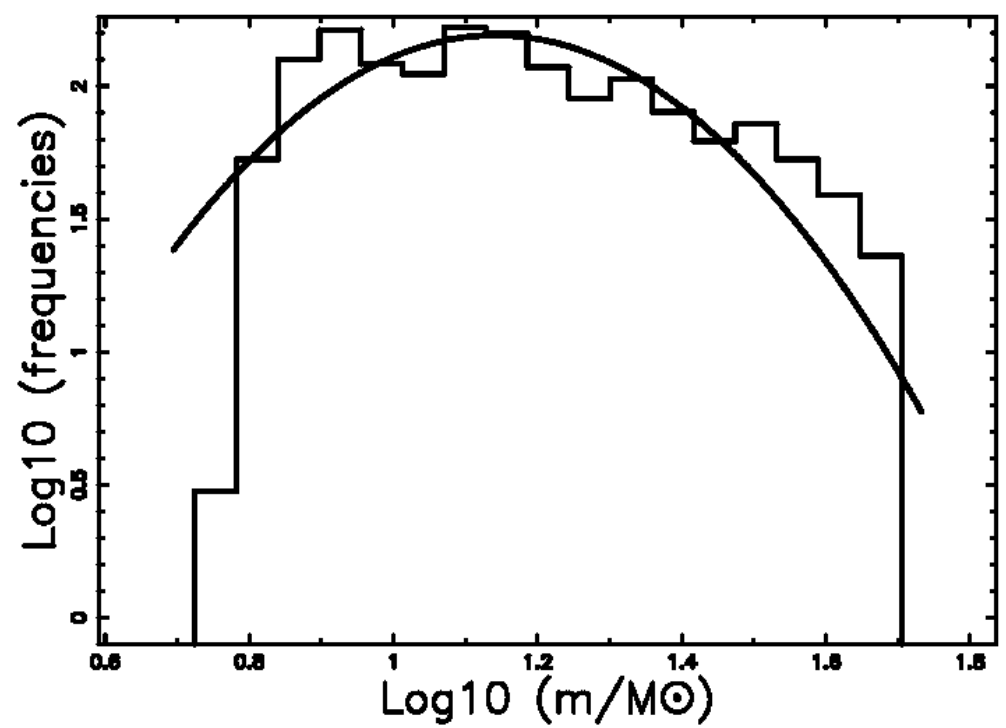

Figure 6. Frequencies of mass distribution for 1563 stars in LMC (steps with full line) with a superposition of the truncated lognormal PDF (full line). Parameters as in Figure 5. The vertical and horizontal axes have a logarithmic scale. 


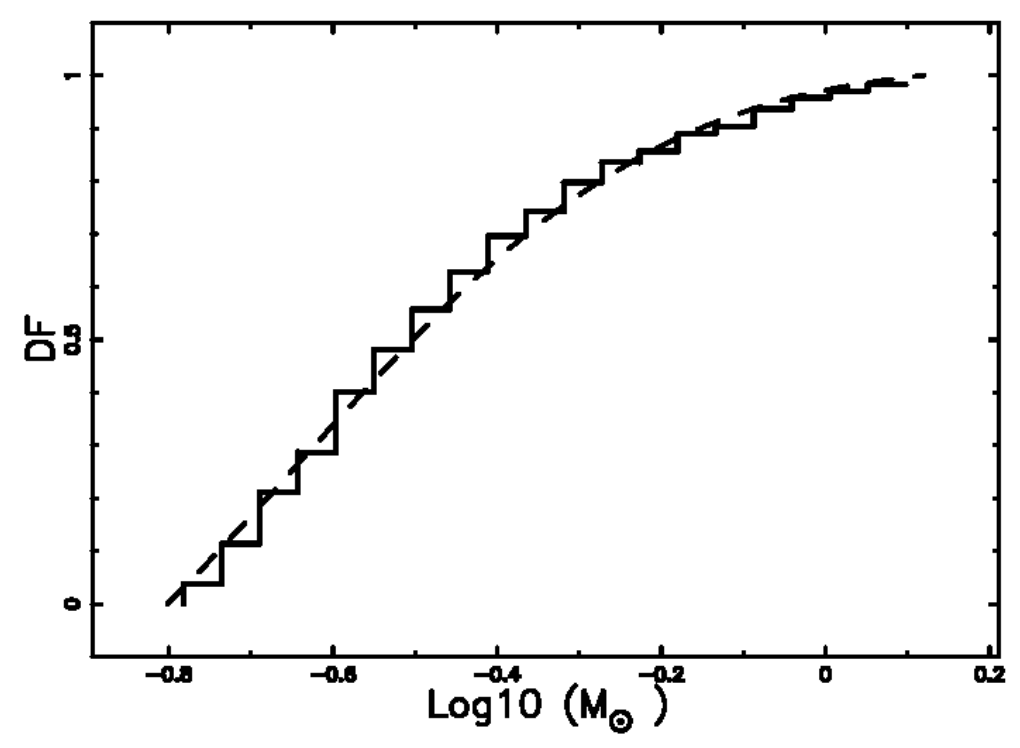

Figure 7. Empirical DF of mass distribution for 237 stars in $\gamma$ Velorum cluster when the number of bins, $n$, is 20 (steps with full line) with a superposition of the truncated lognormal DF (dashed line). Theoretical parameters as in Table 4, MLE method. The horizontal axis has a logarithmic scale.

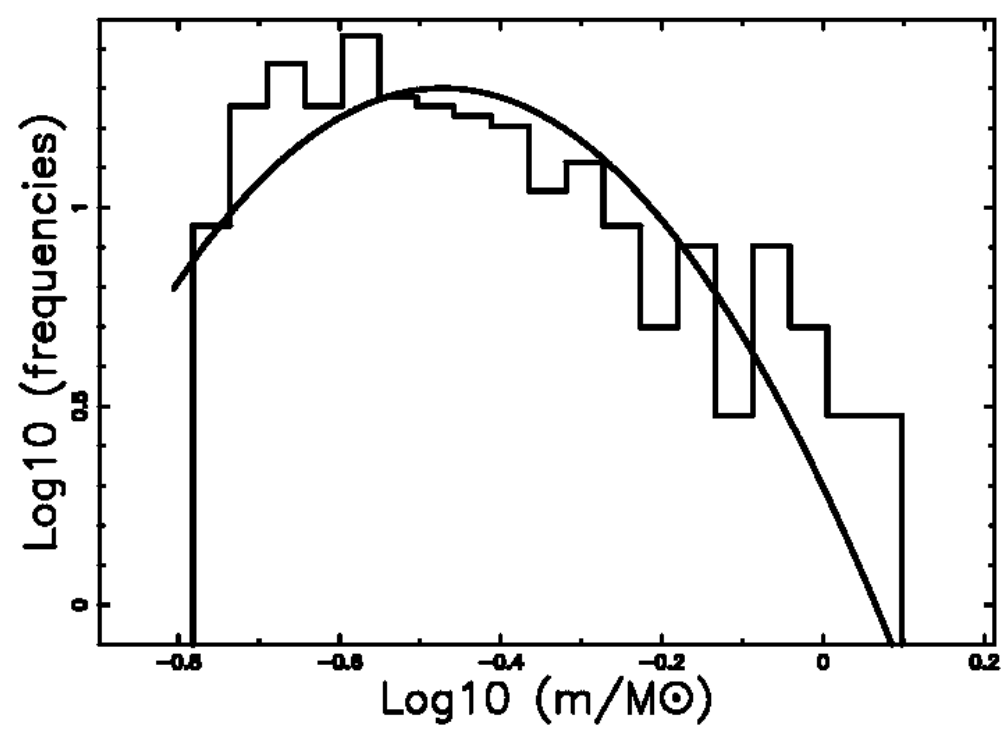

Figure 8. Frequencies of mass distribution for 237 stars in $\gamma$ Velorum cluster (steps with full line) with a superposition of the truncated lognormal PDF (full line). Parameters as in Figure 7. The vertical and horizontal axes have a logarithmic scale. 


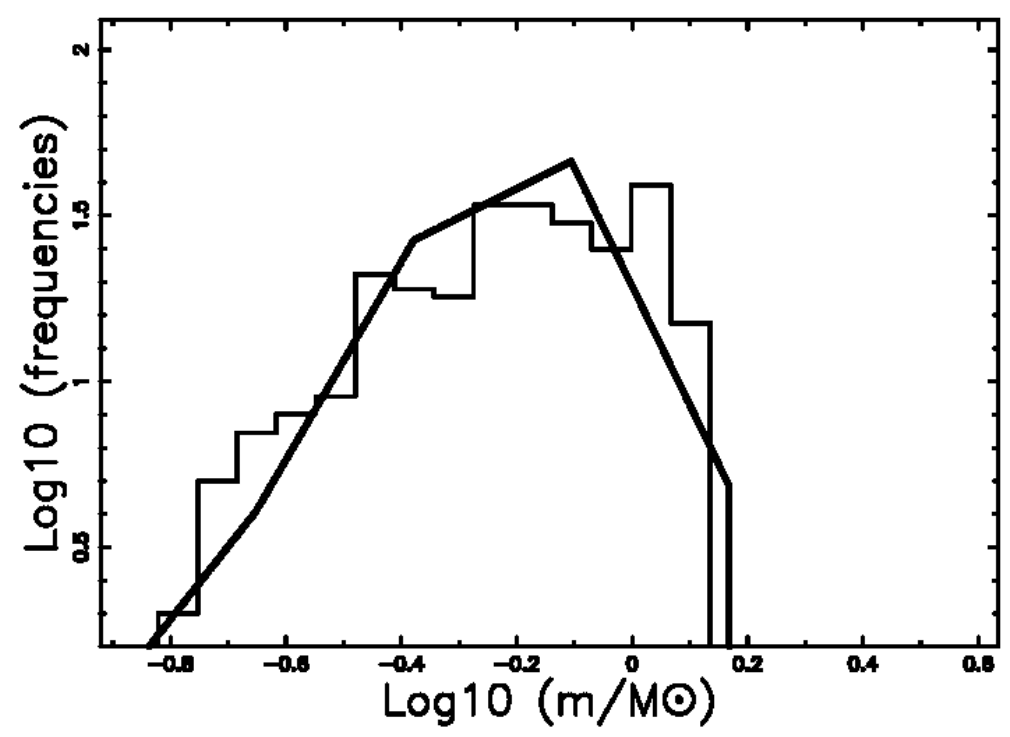

Figure 9. Frequencies of mass distribution for NGC 2362 cluster data (273 stars + BDs) when the number of bins, $n$, is 16 (steps with full line) with a superposition of the four piecewise inverse power law PDF (full line). Theoretical parameters as in Table 5. The vertical and horizontal axes have a logarithmic scale.

We now report three recent PDFs. The first is the double Pareto lognormal distribution which has $\mathrm{PDF}$

$$
\begin{array}{r}
f(x ; \alpha, \beta, \mu, \sigma)=\frac{1}{2} \alpha \beta\left(\mathrm{e}^{\frac{1}{2} \alpha\left(\alpha \sigma^{2}+2 \mu-2 \ln (x)\right)} \operatorname{erfc}\left(\frac{1}{2} \frac{\left(\alpha \sigma^{2}+\mu-\ln (x)\right) \sqrt{2}}{\sigma}\right)\right. \\
\left.+\mathrm{e}^{\frac{1}{2} \beta\left(\beta \sigma^{2}-2 \mu+2 \ln (x)\right)} \operatorname{erfc}\left(\frac{1}{2} \frac{\left(\beta \sigma^{2}-\mu+\ln (x)\right) \sqrt{2}}{\sigma}\right)\right) x^{-1}(\alpha+\beta)^{-1},
\end{array}
$$

where $\alpha$ and $\beta$ are the Pareto coefficients for the upper and the lower tail, respectively, $\mu$ and $\sigma$ are the lognormal body parameters, and erfc is the complementary error function, see [29]. The mean ( for $\alpha>1$ ) can be expressed as

$$
E(\alpha, \beta, \mu, \sigma)=\frac{\alpha \beta \mathrm{e}^{\mu+\frac{1}{2} \sigma^{2}}}{(\alpha-1)(\beta+1)} .
$$

This PDF exhibits a power law behaviour in both tails

$$
f(x) \sim k_{1} x^{-\alpha-1}(x \rightarrow \infty) \quad ; \quad f(x) \sim k_{2} x^{\beta-1}(x \rightarrow 0) \quad,
$$

where $k_{1}$ and $k_{2}$ are two constants. Figures 10 and 11 report the double Pareto lognormal DF and PDF respectively.

The second is the left truncated beta with scale PDF which is

$$
f_{T}(x ; a, b, \alpha, \beta)=K x^{\alpha-1}(b-x)^{\beta-1},
$$

where the constant is

$$
K=\frac{-\alpha \Gamma(\alpha+\beta)}{b^{\beta-1} H a^{\alpha} \Gamma(\alpha+\beta)-b^{\beta-1+\alpha} \Gamma(1+\alpha) \Gamma(\beta)},
$$

and

$$
H={ }_{2} \mathrm{~F}_{1}\left(\alpha,-\beta+1 ; 1+\alpha ; \frac{a}{b}\right),
$$

where ${ }_{2} F_{1}(a, b ; c ; z)$ is the regularized hypergeometric function [30], see [8]. Figure 12 reports the DF and Figure 13 the PDF. 


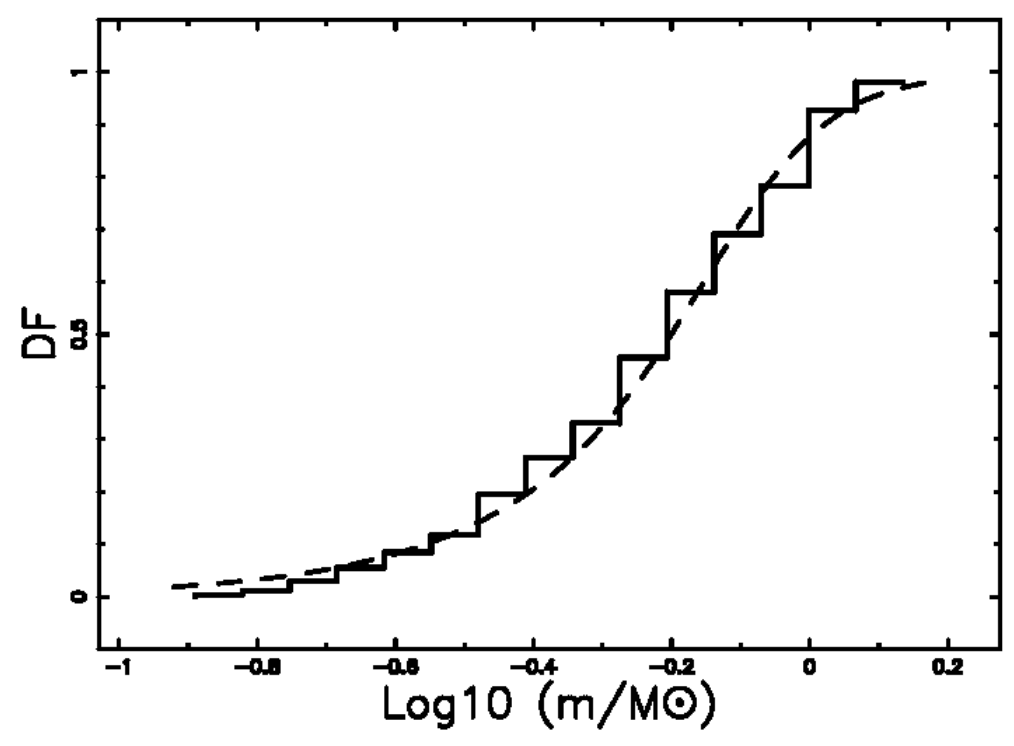

Figure 10. Empirical DF of mass distribution for NGC 2362 cluster data (273 stars + BDs) when the number of bins, $n$, is 16 (steps with full line) with a superposition of the double Pareto lognormal DF (full line). Theoretical parameters as in Table 5. The horizontal axis has a logarithmic scale.

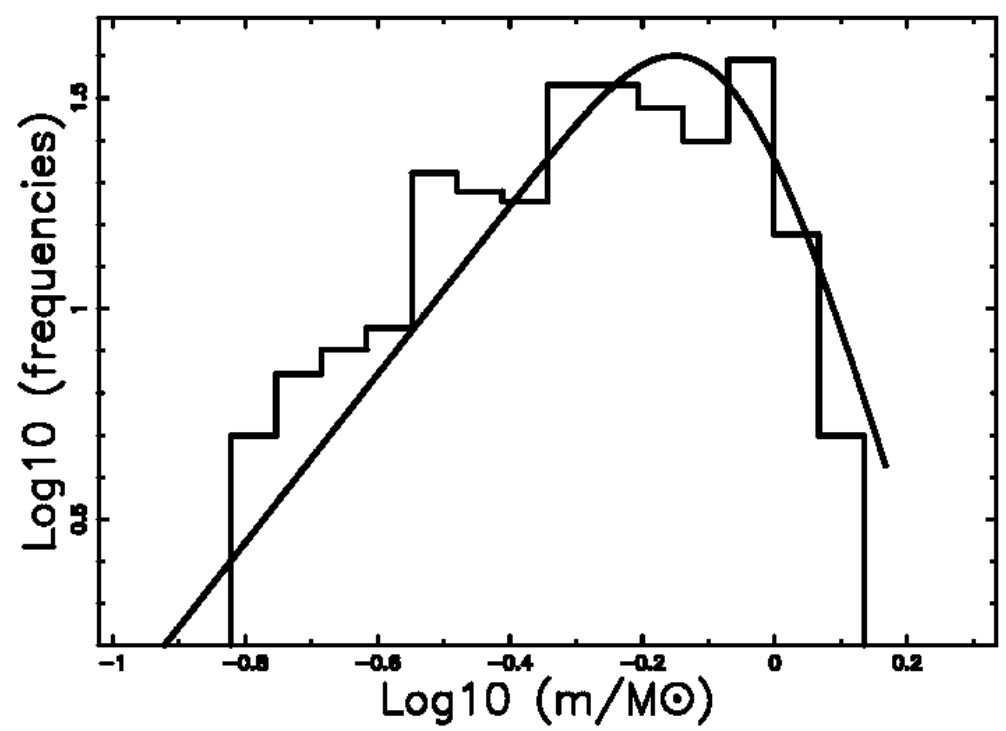

Figure 11. Frequencies of mass distribution for NGC 2362 cluster data ( 273 stars + BDs) when the number of bins, $n$, is 16 (steps with full line) with a superposition of the double Pareto lognormal PDF (full line). Theoretical parameters as in Table 5. The vertical and horizontal axes have a logarithmic scale. 


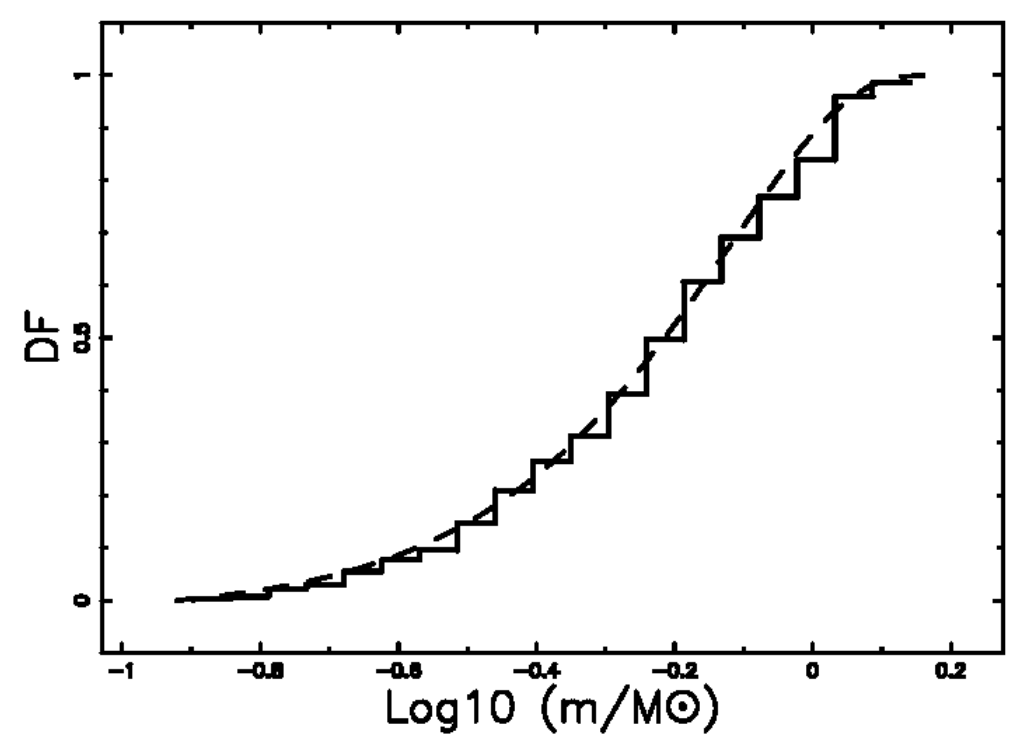

Figure 12. Empirical DF of mass distribution for NGC 2362 cluster data ( 273 stars + BDs) when the number of bins, $n$, is 20 (steps at full line) with a superposition of the left truncated beta DF (full line). Theoretical parameters as in Table 5. The horizontal axis has a logarithmic scale.

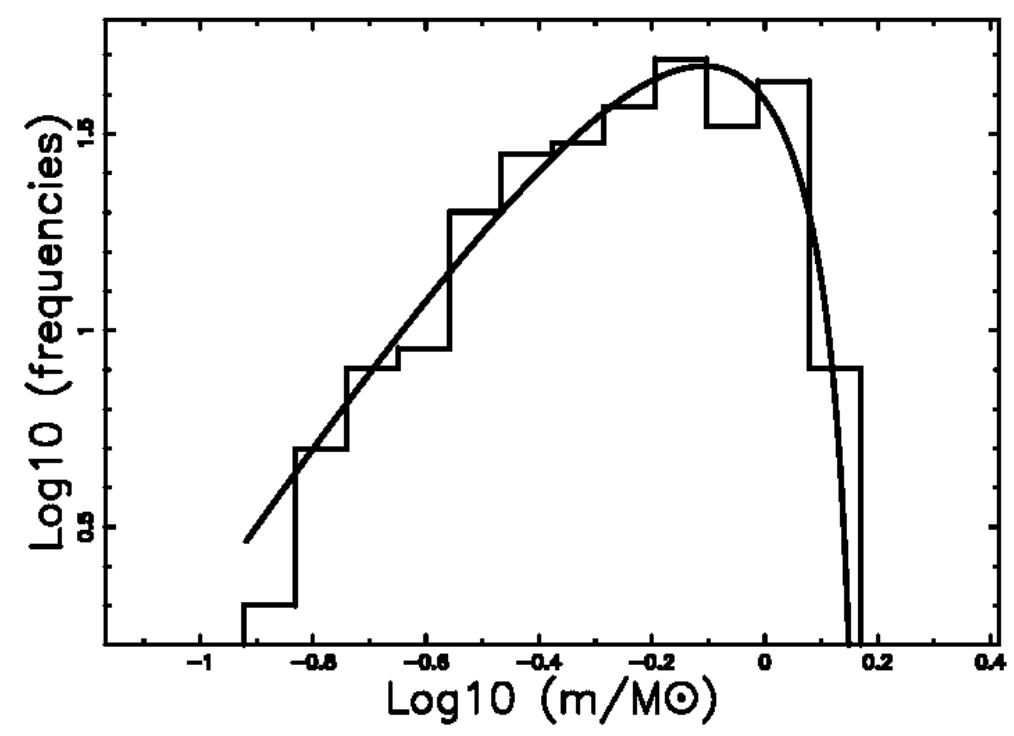

Figure 13. Frequencies for mass distribution in NGC 2362 cluster with (full line steps) and left truncated beta PDF. Parameters as in Figure 12. The vertical and horizontal axes have a logarithmic scale. 
The third is the truncated gamma (TG) PDF which is

$$
f\left(x ; b, c, x_{l}, x_{u}\right)=k\left(\frac{x}{b}\right)^{c-1} \mathrm{e}^{-\frac{x}{b}}
$$

where the constant $k$ is

$$
k=\frac{c}{b \Gamma\left(1+c, \frac{x_{l}}{b}\right)-b \Gamma\left(1+c, \frac{x_{u}}{b}\right)+\mathrm{e}^{-\frac{x_{u}}{b}} b^{-c+1} x_{u}^{c}-\mathrm{e}^{-\frac{x_{l}}{b}} b^{-c+1} x_{l}{ }^{c}},
$$

where

$$
\Gamma(a, z)=\int_{z}^{\infty} t^{a-1} e^{-t} \mathrm{~d} t
$$

is the upper incomplete gamma function, see [31].

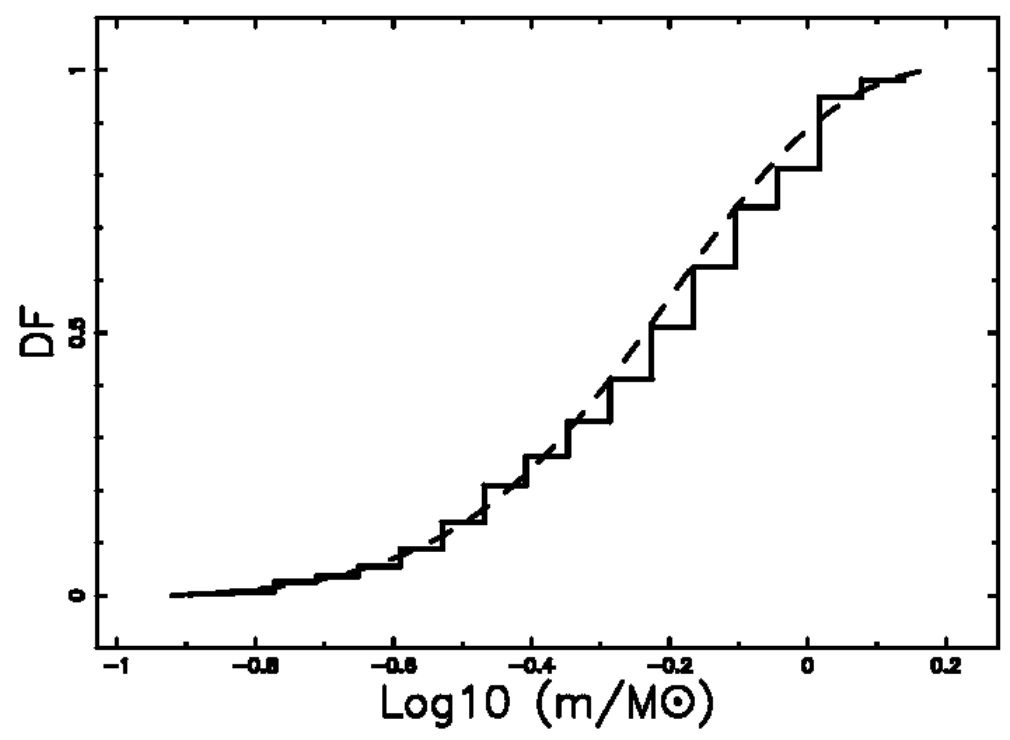

Figure 14. Empirical DF of mass distribution for NGC 2362 cluster data ( 273 stars + BDs) when the number of bins, $n$, is 18 (steps at full line) with a superposition of the truncated gamma DF (full line). Theoretical parameters as in Table 5 . The horizontal axis has a logarithmic scale.

Figure 14 reports the truncated gamma DF and Figure 15 the truncated gamma PDF. Table 5 reports the parameters of these three new PDFs as well as the parameters of the truncated lognormal in the case of NGC 2362. can be found Figure 16 displays all the PDFs here analysed.

Table 5. Statistical parameters of NGC 2362 (272 stars) for different distributions

\begin{tabular}{|c|c|c|c|}
\hline PDF & parameters & $\mathrm{D}$ & $P_{K S}$ \\
\hline truncated lognormal & $\sigma=0.59, m=0.625, x_{l}=0.119, x_{u}=1.47$ & 0.047 & 0.556 \\
\hline truncated gamma & $b=0.159, c=4, x_{l}=0.12, x_{u}=1.47$ & 0.067 & 0.158 \\
\hline double Pareto-lognormal & $\alpha=5, \beta=2, \sigma=0.207, \mu_{L N}=-0.25$ & 0.05 & 0.471 \\
\hline left truncated beta & $a=0.12, b=1.47, \alpha=2.18, \beta=2.93$ & 0.048 & 0.53 \\
\hline four inverse power law & $m_{1}=0.11, m_{2}=0.22, m 3=0.41, m_{4}=0.78, m_{5}=1.47$ & 0.081 & 0.052 \\
& $\alpha_{1}=-1.2, \alpha_{2}=-1.98, \alpha_{3}=0.12, \alpha_{4}=4.57$ & & \\
\hline
\end{tabular}




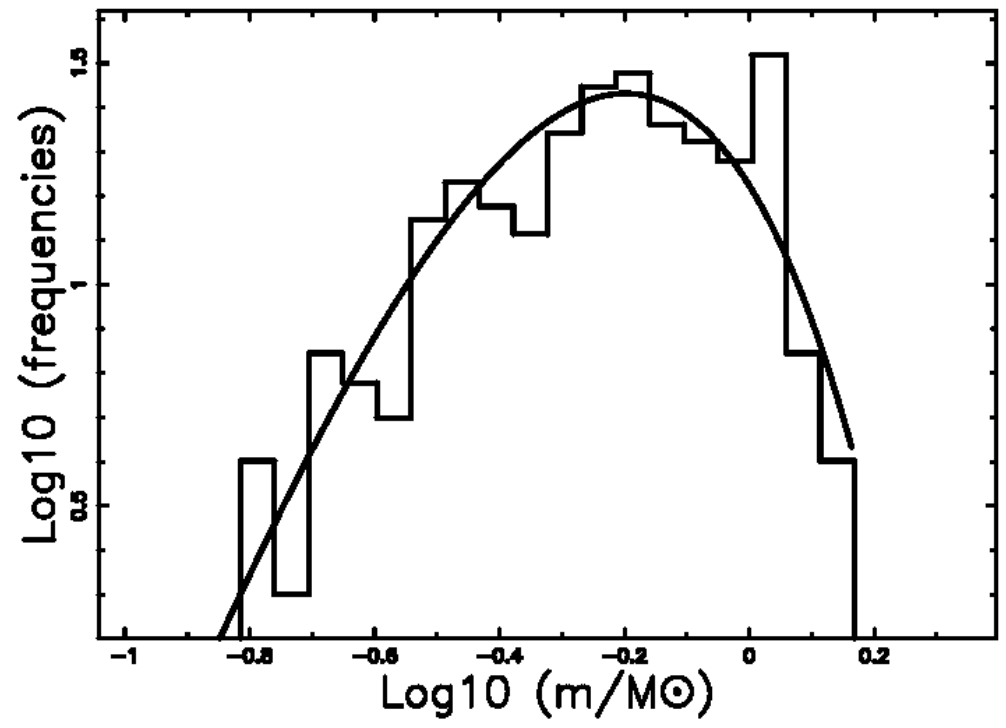

Figure 15. Frequencies of mass distribution for NGC 2362 cluster data (273 stars + BDs) when the number of bins, $n$, is 18 (steps at full line) with a superposition of the truncated gamma PDF (full line). Theoretical parameters as in Table 5. The vertical and horizontal axes have a logarithmic scale.

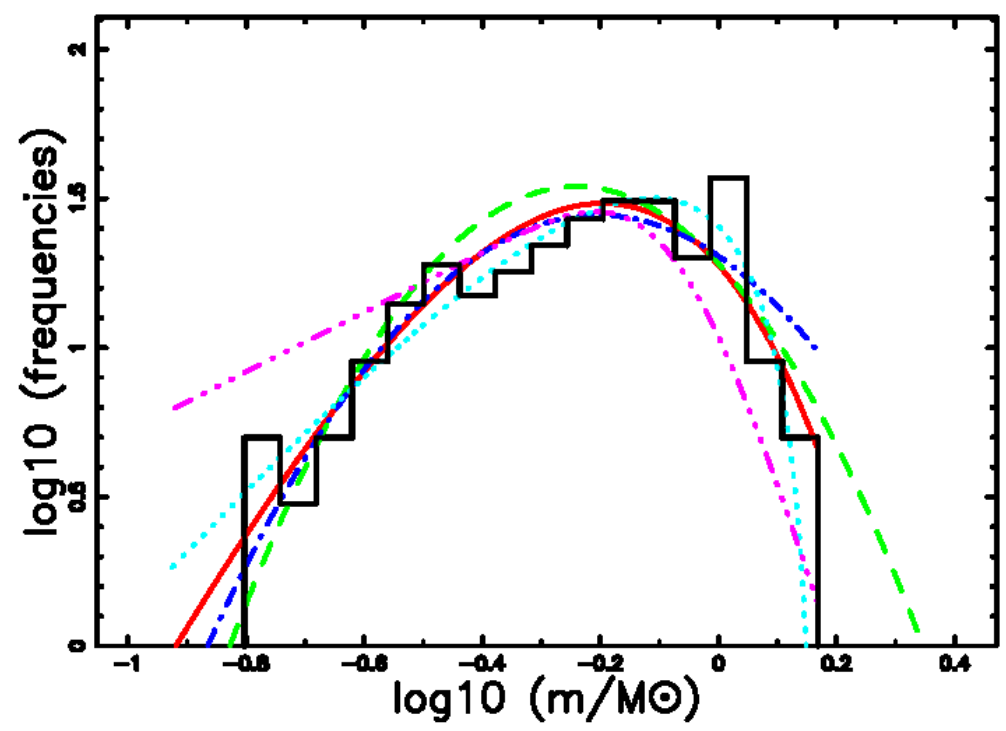

Figure 16. Histogram (black step-diagram) of mass distribution as given by NGC 2362 cluster data (273 stars +BDs ) with a superposition of the truncated gamma PDF (full red line), the lognormal PDF (dashed green line ), the truncated lognormal PDF (dot-dash-dot-dash blue line), the truncated beta PDF (dotted cyan line) and the double Pareto lognormal PDF (dash-dot-dot-dot magenta line). Vertical and horizontal axes have logarithmic scales. 


\section{Conclusions}

The truncated lognormal distribution gives better results, i.e. higher $P_{K S}$, than the lognormal distribution, see Tables 1, 2, 3 and 4. for the samples here considered. The lower and upper boundaries in mass are connected with the physical theories on the minimum and maximum mass for the stars. Fisher's conjecture (see [32]) that statistical parameters are better inferred through the maximum likelihood estimator (MLE) than through the matching of moments estimator (MME) is also tested: in eight cases out of eight, the MLE produces better results, see Tables 1, 2, 3 and 4. The comparison of the truncated lognormal DF with other DFs assigns the best results to the truncated lognormal, i.e. higher $P_{K S}$, even if the difference from the double Pareto lognormal is small, see Table 5.

The number of free parameters of the truncated lognormal PDF is two once the lower and upper boundary are associated with the minimum and maximum mass of the considered sample, see 6 for the MLE method. In contrast, the number of parameters of the widely used four-piecewise broken inverse power law IMF is seven.

\section{Appendix: The Parameters of the Truncated Lognormal}

The parameters of the truncated lognormal distribution can be obtained from empirical data by the maximum likelihood estimators (MLE) and by the evaluation of the minimum and maximum elements of the sample. Consider a sample $\mathcal{X}=x_{1}, x_{2}, \ldots, x_{n}$ and let $x_{(1)} \geq x_{(2)} \geq \cdots \geq x_{(n)}$ denote their order statistics, so that $x_{(1)}=\max \left(x_{1}, x_{2}, \ldots, x_{n}\right), x_{(n)}=\min \left(x_{1}, x_{2}, \ldots, x_{n}\right)$. The first two parameters $x_{l}$ and $x_{u}$ are

$$
x_{l}=x_{(n)}, \quad x_{u}=x_{(1)} .
$$

The MLE is obtained by maximizing

$$
\Lambda=\sum_{i}^{n} \ln \left(T L\left(x_{i} ; m, \sigma, x_{l}, x_{u}\right)\right) .
$$

The two derivatives $\frac{\partial \Lambda}{\partial m}=0$ and $\frac{\partial \Lambda}{\partial \sigma}=0$ generate two non-linear equations in $m$ and $\sigma$ which can be solved numerically, we used FORTRAN subroutine SNSQE in [17],

$$
\begin{array}{r}
\frac{\partial \Lambda}{\partial m}=\left(\operatorname{erf}\left(\frac{1}{2} \frac{\sqrt{2}\left(\ln \left(x_{l}\right)-\ln (m)\right)}{\sigma}\right)-\operatorname{erf}\left(\frac{1}{2} \frac{\sqrt{2}\left(\ln \left(x_{u}\right)-\ln (m)\right)}{\sigma}\right)\right) \\
\left(n \sqrt{2} \sigma \mathrm{e}^{-\frac{1}{2} \frac{\left(\ln \left(x_{l}\right)-\ln (m)\right)^{2}}{\sigma^{2}}}-n \sqrt{2} \sigma \mathrm{e}^{-\frac{1}{2} \frac{\left(\ln \left(x_{u}\right)-\ln (m)\right)^{2}}{\sigma^{2}}}\right. \\
-\sqrt{\pi}\left(\operatorname{erf}\left(\frac{1}{2} \frac{\sqrt{2}\left(\ln \left(x_{l}\right)-\ln (m)\right)}{\sigma}\right)\right. \\
\left.\left.-\operatorname{erf}\left(\frac{1}{2} \frac{\sqrt{2}\left(\ln \left(x_{u}\right)-\ln (m)\right)}{\sigma}\right)\right)\left(n \ln (m)-\sum_{i=1}^{n} \ln \left(x_{i}\right)\right)\right)=0
\end{array}
$$

and

$$
\frac{\partial \Lambda}{\partial \sigma}=\frac{N}{D}=0
$$


where

$$
\begin{aligned}
& N=\ln \left(x_{u}\right) \sqrt{2} \mathrm{e}^{-\frac{1}{2} \frac{\left(\ln \left(x_{u}\right)-\ln (m)\right)^{2}}{\sigma^{2}}} n \sigma-\ln \left(x_{l}\right) \sqrt{2} \mathrm{e}^{-\frac{1}{2} \frac{\left(\ln \left(x_{l}\right)-\ln (m)\right)^{2}}{\sigma^{2}}} n \sigma \\
& +\sqrt{2} \mathrm{e}^{-1 / 2 \frac{\left(\ln \left(x_{l}\right)-\ln (m)\right)^{2}}{\sigma^{2}}} \ln (m) n \sigma-\sqrt{2} \mathrm{e}^{-\frac{1}{2} \frac{\left(\ln \left(x_{u}\right)-\ln (m)\right)^{2}}{\sigma^{2}}} \ln (m) n \sigma \\
& +n(\ln (m))^{2} \sqrt{\pi} \operatorname{erf}\left(\frac{1}{2} \frac{\sqrt{2}\left(\ln \left(x_{u}\right)-\ln (m)\right)}{\sigma}\right) \\
& -n \sigma^{2} \sqrt{\pi} \operatorname{erf}\left(\frac{1}{2} \frac{\sqrt{2}\left(\ln \left(x_{u}\right)-\ln (m)\right)}{\sigma}\right) \\
& -n(\ln (m))^{2} \sqrt{\pi} \operatorname{erf}\left(\frac{1}{2} \frac{\sqrt{2}\left(\ln \left(x_{l}\right)-\ln (m)\right)}{\sigma}\right) \\
& +n \sigma^{2} \sqrt{\pi} \operatorname{erf}\left(\frac{1}{2} \frac{\sqrt{2}\left(\ln \left(x_{l}\right)-\ln (m)\right)}{\sigma}\right) \\
& +\sum_{i=1}^{n} \ln \left(x_{i}\right)\left(\ln \left(x_{i}\right)-2 \ln (m)\right) \sqrt{\pi} \operatorname{erf}\left(\frac{1}{2} \frac{\sqrt{2}\left(\ln \left(x_{u}\right)-\ln (m)\right)}{\sigma}\right) \\
& -\sum_{i=1}^{n} \ln \left(x_{i}\right)\left(\ln \left(x_{i}\right)-2 \ln (m)\right) \sqrt{\pi} \operatorname{erf}\left(\frac{1}{2} \frac{\sqrt{2}\left(\ln \left(x_{l}\right)-\ln (m)\right)}{\sigma}\right), \\
& D=\sqrt{\pi}\left(-\operatorname{erf}\left(\frac{1}{2} \frac{\sqrt{2}\left(\ln \left(x_{l}\right)-\ln (m)\right)}{\sigma}\right)\right. \\
& \left.+\operatorname{erf}\left(\frac{1}{2} \frac{\sqrt{2}\left(\ln \left(x_{u}\right)-\ln (m)\right)}{\sigma}\right)\right) \sigma^{3} \text {. }
\end{aligned}
$$

\section{References}

1. E. E. Salpeter, The Luminosity Function and Stellar Evolution., ApJ 121 (1955) 161-167.

2. J. M. Scalo, The stellar initial mass function, Fundamentals of Cosmic Physics 11 (1986) 1-278.

3. P. Kroupa, C. A. Tout, G. Gilmore, The distribution of low-mass stars in the Galactic disc, MNRAS 262 (1993) 545-587.

4. J. Binney, M. Merrifield, Galactic astronomy, Princeton University Press, Princeton, NJ, 1998.

5. P. Kroupa, On the variation of the initial mass function, MNRAS 322 (2001) 231-246.

6. N. Bastian, K. R. Covey, M. R. Meyer, A Universal Stellar Initial Mass Function? A Critical Look at Variations, ARA\&A 48 (2010) 339-389. arXiv:1001.2965, doi:10.1146/annurev-astro-082708-101642.

7. P. Kroupa, C. Weidner, J. Pflamm-Altenburg, I. Thies, J. Dabringhausen, M. Marks, T. Maschberger, The Stellar and Sub-Stellar Initial Mass Function of Simple and Composite Populations, Springer Netherlands, 2013, p. 115.

8. L. Zaninetti, The initial mass function modeled by a left truncated beta distribution , ApJ 765 (2013) 128-135.

9. M. Evans, N. Hastings, B. Peacock, Statistical Distributions - third edition, John Wiley \& Sons Inc, New York, 2000.

10. R. B. Larson, A simple probabilistic theory of fragmentation, MNRAS 161 (1973) 133. doi:10.1093/mnras/ 161.2 .133

11. G. E. Miller, J. M. Scalo, The initial mass function and stellar birthrate in the solar neighborhood, ApJS 41 (1979) 513-547. doi:10.1086/190629.

12. H. Zinnecker, Star formation from hierarchical cloud fragmentation - A statistical theory of the log-normal Initial Mass Function, MNRAS 210 (1984) 43-56. doi:10.1093/mnras/210.1.43.

13. G. Chabrier, Galactic Stellar and Substellar Initial Mass Function, PASP 115 (2003) 763-795. arXiv:arXiv: astro-ph/0304382, doi:10.1086/376392.

14. A. N. Cox, Allen's astrophysical quantities, Springer, New York, 2000.

15. N. L. Johnson, S. Kotz, N. Balakrishnan, Continuous univariate distributions. Vol. 1. 2nd ed., Wiley , New York, 1994. 
16. F. W. J. e. Olver, D. W. e. Lozier, R. F. e. Boisvert, C. W. e. Clark, NIST handbook of mathematical functions., Cambridge University Press. , Cambridge, 2010.

17. D. Kahaner, C. Moler, S. Nash, Numerical Methods and Software, Prentice Hall Publishers, Englewood Cliffs, New Jersey, 1989.

18. W. H. Press, S. A. Teukolsky, W. T. Vetterling, B. P. Flannery, Numerical Recipes in FORTRAN. The Art of Scientific Computing, Cambridge University Press, Cambridge, UK, 1992.

19. H. Akaike, A new look at the statistical model identification, IEEE Transactions on Automatic Control 19 (1974) 716-723.

20. A. R. Liddle, How many cosmological parameters?, MNRAS 351 (2004) L49-L53.

21. W. Godlowski, M. Szydowski, Constraints on Dark Energy Models from Supernovae, in: M. Turatto, S. Benetti, L. Zampieri, W. Shea (Eds.), 1604-2004: Supernovae as Cosmological Lighthouses, Vol. 342 of Astronomical Society of the Pacific Conference Series, 2005, pp. 508-516.

22. A. Kolmogoroff, Confidence limits for an unknown distribution function, The Annals of Mathematical Statistics 12 (4) (1941) 461-463.

23. N. Smirnov, Table for estimating the goodness of fit of empirical distributions, The Annals of Mathematical Statistics 19 (2) (1948) 279-281.

24. J. Massey, Frank J., The kolmogorov-smirnov test for goodness of fit, Journal of the American Statistical Association 46 (253) (1951) 68-78.

25. J. M. Oliveira, R. D. Jeffries, J. T. van Loon, The low-mass initial mass function in the young cluster NGC 6611, MNRAS 392 (2009) 1034-1050. arXiv:0810.4444, doi:10.1111/j.1365-2966.2008.14140.x.

26. J. Irwin, S. Hodgkin, S. Aigrain, J. Bouvier, L. Hebb, M. Irwin, E. Moraux, The Monitor project: rotation of low-mass stars in NGC 2362 - testing the disc regulation paradigm at 5 Myr, MNRAS 384 (2008) 675-686. arXiv:0711.2398, doi:10.1111/j.1365-2966.2007.12725.x.

27. J. K. Hill, J. E. Isensee, R. H. Cornett, R. C. Bohlin, R. W. O'Connell, M. S. Roberts, A. M. Smith, T. P. Stecher, Initial mass functions from ultraviolet stellar photometry: A comparison of Lucke and Hodge OB associations near 30 Doradus with the nearby field, ApJ 425 (1994) 122-126. doi:10.1086/173968.

28. L. Prisinzano, F. Damiani, G. Micela, R. D. Jeffries, E. Franciosini, G. G. Sacco, A. Frasca, A. Klutsch, A. Lanzafame, E. J. Alfaro, K. Biazzo, R. Bonito, A. Bragaglia, M. Caramazza, A. Vallenari, G. Carraro, M. T. Costado, E. Flaccomio, P. Jofré, C. Lardo, L. Monaco, L. Morbidelli, N. Mowlavi, E. Pancino, S. Randich, S. Zaggia, The Gaia-ESO Survey: membership and initial mass function of the $\gamma$ Velorum cluster, A\&A 589 (2016) A70. arXiv:1601.06513, doi:10.1051/0004-6361/201527875.

29. W. J. Reed, M. Jorgensen, The double pareto-lognormal distributiona new parametric model for size distributions, Communications in Statistics - Theory and Methods 33 (8) (2004) 1733-1753. arXiv:http: //www.tandfonline.com/doi/pdf/10.1081/STA-120037438, doi:10.1081/STA-120037438. URL http://www.tandfonline.com/doi/abs/10.1081/STA-120037438

30. M. Abramowitz, I. A. Stegun, Handbook of Mathematical Functions with Formulas, Graphs, and Mathematical Tables, Dover, New York, 1965.

31. L. Zaninetti, A right and left truncated gamma distribution with application to the stars, Advanced Studies in Theoretical Physics 23 (2013) 1139-1147.

32. A. Hald, On the history of maximum likelihood in relation to inverse probability and least squares, Statist. Sci. 14 (2) (1999) 214-222. doi:10.1214/ss/1009212248.

URL http://dx.doi.org/10.1214/ss/1009212248 